\title{
La productividad académica en las instituciones de educación superior en México: de la teoría a la práctica
}

\section{Academic productivity in higher education in Mexico: from theory to practice}

\author{
Jessica M. Gordillo-Salazar \\ Universidad Autónoma de Estado de Hidalgo, México \\ ORCID: https://orcid.org/0000-0002-2899-8250 \\ Yolanda Sánchez-Torres \\ Universidad Autónoma del Estado de Hidalgo, México \\ ORCID: http://orcid.org/0000-0002-7372-6123 \\ Aníbal Terrones-Cordero \\ Universidad Autónoma del Estado de Hidalgo, México \\ ORCID: http://orcid.org/0000-0002-2959-1807 \\ Mario Cruz-Cruz \\ Universidad Autónoma del Estado de Hidalgo, México \\ ORCID: https://orcid.org/0000-0003-1399-9223
}

Received 03-24-20 Revised 05-30-20 Accepted 07-07-20 On line 12-04-20

*Correspondence

Email: yolasato08@hotmail.com
Cite as:

Gordillo-Salazar, J., Sánchez-Torres, Y., Terrones-Cordero, A., \& Cruz-Cruz, M. (2020). La productividad académica en las instituciones de educación superior en México: de la teoría a la práctica. Propósitos y Representaciones, 8(3), e441. doi: http://dx.doi.org/10.20511/pyr2020.v8n3.441 


\section{Resumen}

La productividad es uno de los conceptos básicos de las ciencias económicas, entendido en los términos más simples como una relación entre los insumos (inputs) y los productos (outputs), asociada generalmente a las organizaciones productoras de bienes. Sin embargo, la nueva economía, apoyada en el sector terciario, tiene su principal fortaleza en aquellas organizaciones de servicios basados en conocimiento (SBC), como es la educación, en donde la productividad se torna en algo más complejo de explicar. Es por ello que la finalidad del presente documento es argumentar sobre la inconsistencia del concepto de productividad académica en las instituciones de educación superior en México, a través de una revisión documental teórica y empírica. La productividad académica en el caso de México, está vinculada a cuatro actividades que realizan los profesores de tiempo completo: docencia, tutoría, gestión académica y generación y aplicación del conocimiento, esto último de acuerdo al Programa para el Desarrollo Profesional Docente en Educación Superior. La evidencia empírica, tanto para México como a nivel internacional, muestra como la productividad académica se limita a estudiar las actividades relacionadas con la generación y aplicación del conocimiento, pocos incluyen docencia y prácticamente ninguno a la tutoría y gestión académica. El documento evidencia como la productividad académica no está definida conceptual, ni normativamente, de tal forma que no se puede establecer una valoración objetiva entre instituciones, países o regiones.

Palabras clave: productividad académica, sector servicios, profesores de tiempo completo, educación superior, México.

\section{Summary}

Productivity is one of the basic concepts of economic sciences, is well-known as a relationship between supplies (inputs) and products (outputs), generally associated with goods-producing organizations. However, the new economy, supported by the tertiary sector has its main strength in those knowledge-based service organizations (SBC) such as education, where productivity becomes more complex to explain. The purpose of this document is to provide an overview of the divergency about the concept of academic productivity in higher education institutions in Mexico, through a theoretical and empirical documentary review. Academic productivity in Mexico is linked to four activities carried out by full-time teachers: teaching, tutoring, academic management and knowledge generation and application, the latter according to the Program for the Professional Development of Teachers in Higher Education. As a result in empirical evidence, not just for Mexico, shows how academic productivity is limited to studying activities related to the generation and application of knowledge, on the other hand, few incorporate teaching and none to tutoring and academic management. The document shows how academic productivity is not conceptually defined, nor normatively; as a result of it an objective assessment between institutions, countries or regions cannot be established.

Keywords: academic productivity, service sector, full-time teachers, higher education; Mexico.

\section{Introducción}

El concepto de productividad surgió a mediados del siglo XVIII, con el paso del tiempo fue cobrando relevancia dentro del contexto económico de un país y de toda organización. De acuerdo con Velásquez de Naime, Rodríguez \& Guaita (2012), en la actualidad, toda nación que presuma 
ser competitiva pone especial interés en buscar las diferentes vías que permitan generar y sostener altos niveles de productividad, por lo que se convierte en un determinante fundamental del desempeño de toda economía (Domínguez, 2017). De acuerdo a lo anterior, se puede decir que la productividad permite identificar la eficiencia en el uso de los recursos con los que se cuenta. Cabe destacar que en general este concepto está enfocado a la producción de bienes y a pesar de que resulta complicado ponerlo en práctica en una organización de servicios, es posible su análisis, siempre y cuando se adapte a las características propias de la actividad que se desarrolla.

Labra (2003) y Maroto (2013), refieren que en la actualidad no se puede negar que existe un entorno más competitivo para el sector de servicios; esto puede ser consecuencia de la importancia que está tomando; sobre todo por su amplia presencia en los procesos productivos y de integración ente los mercados y la globalización de las economías desarrolladas, aunado a la generación de empleos y el peso significativo en el Producto Interno Bruto (PIB) (López, Niembro \& Ramos, 2017). Por lo anterior, el concepto de productividad queda corto cuando refiere a organizaciones de servicios o terciarias ya que no cubre con las necesidades propias de esta tipología de organización.

La nueva economía, basada en el sector terciario, tiene su principal fortaleza en aquellas organizaciones de servicios basados en conocimiento (SBC), la cuales pueden desarrollar productos relacionados con la educación, salud, ingeniería, software o investigación y desarrollo; ejemplo de esto, son países como China, India e Irlanda quienes están inclinando su economía a formar ciudadanos con educación superior (López et al., 2017).

La trascendencia de la educación, vista desde la perspectiva de mercancía, hace obligatorio realizar un estudio sobre la productividad en los centros de educación superior, ya que de ellas emergen nuevos conocimientos, que en muchos casos se traducen en innovaciones que benefician a la sociedad (Labra, 2003).

A lo anterior se le puede agregar la naturaleza intangible de las organizaciones de servicios, que hace más complejo el poder tener una definición única sobre la productividad en organizaciones prestadoras de servicios. En consecuencia, el objetivo de la presente investigación fue presentar una argumentación teórica sobre el concepto de productividad académica en educación superior en México; la cual, tiene como fin último la generación de productividad cognitiva y no simplemente la de generar ganancias económicas (Narváez \& Burgos, 2011; Munévar \& Villaseñor, 2008).

La discusión teórica sobre la productividad se hizo a través de una revisión extensa referida a la literatura actual sobre el concepto de productividad, acotada a las organizaciones prestadoras de servicios de la educación superior en México.

Durante el proceso de búsqueda se pudo identificar que la productividad académica tiene un mayor peso en la educación superior, sobre todo si es una educación de calidad porque su impacto se ve reflejado en temas como equidad de género, crecimiento económico, bienestar, producción y cambio climático, según la Organización de las Naciones Unidas para la Educación, la Ciencia y la Cultura (UNESCO, 2017). Lo anterior es clave para el desarrollo económico de un país, ya que al contar con un capital humano con mayor conocimiento permite ser más competitivo, innovador, entre otros beneficios (Llisterri, Gligo, Homs \& Ruíz-Devesa, 2014).

La productividad académica en educación superior se encuentra vinculada a las actividades que realizan los Profesores de Tiempo Completo (PTC). Dentro de estas, destacan 
docencia e investigación (Martínez \& Coronado, 2003; Carayol \& Matt, 2006; Amado-Moreno, Sevilla-García, Galaz-Fontes \& Brito-Páez, 2015 ). Aunque es esta última la que lleva el rol predominante dentro de la literatura (Dundar \& Lewis, 1998; García-Cepero, 2010; Martínez \& Sánchez, 2014).

En México, el organismo encargado de delimitar las actividades de los PTC, en las Instituciones de Educación Superior (IES), es la Secretaria de Educación Pública (2018), mediante el Programa para el Desarrollo Profesional Docente en Educación Superior (Prodep), quien establece cuatro tipo de funciones: docencia, tutorías y dirección individualizada del alumno, gestión académica y generación o aplicación innovadora del conocimiento.

La hipótesis que se postula es que en México la productividad académica en educación superior presenta un desfase entre lo que señala la literatura y lo que establece la parte normativa que las autoridades mexicanas estipulan. De esta forma es difícil evaluar objetivamente esta productividad en demerito de su capacidad competitiva, comparada con otras IES, dentro y fuera de México.

Para un mejor entendimiento sobre la argumentación del presente documento. Se presenta en primera instancia una revisión histórica sobre el concepto de la productividad. Posteriormente; se retoma la productividad vista desde la perspectiva de las organizaciones de servicio o terciarias, en específico aquellas dedicadas a la educación, de tal forma que lo anterior permita aproximarse al término de productividad académica, sus indicadores y los factores internos y externos que la impactan. Por último, se presentan las conclusiones y referencias bibliográficas.

\section{Argumentación}

\section{Sobre el concepto de productividad}

Actualmente las investigaciones refieren que para estar en posibilidades de contender dentro del mercado internacional es importante desarrollar productos o servicios competitivos, esto puede ser posible mediante la optimización de los recursos, lo cual implica, aumentar los niveles de productividad (Bonilla, 2014).

De acuerdo con Sumanth (1999), es Quesnay quien menciona por primera vez el término de productividad, entendido como aquel elemento fundamental que permite obtener más con menor esfuerzo. Es a partir de este concepto cuando la productividad comienza a ser parte de las discusiones dentro del pensamiento económico, Con el paso del tiempo se ha convertido en una pieza clave para el progreso de las sociedades y un componente imprescindible de la economía de un país (Domínguez, 2017).

En la actualidad es inobjetable la relevancia de la productividad dentro del ámbito económico sobre todo por el impacto que genera en el nivel de vida de los individuos (Martínez, 2017). Sin embargo, este concepto, como muchos otros, se ha ido construyendo a través del tiempo, tal y como se muestra en la Tabla 1, donde se presenta una revisión histórica por diversos autores que abonan sobre este concepto. 
Tabla 1.

Evolución del concepto productividad a través del tiempo

\begin{tabular}{lll} 
Autores & Año & Definición \\
\hline Early & 1900 & $\begin{array}{l}\text { "Relación entre producción y los medio empleados para } \\
\text { lograrla" (Sumanth, 1990, p. 2). }\end{array}$ \\
\hline OCEE & 1950 & $\begin{array}{l}\text { "Cociente que se obtiene al dividir la producción por uno de los } \\
\text { factores de producción" (Sumanth, 1990, p. 2). }\end{array}$
\end{tabular}

\begin{tabular}{lll}
\hline Fabricant 1962 & $\begin{array}{l}\text { "Siempre-una razón entre la producción y los insumos" } \\
\text { (Sumanth, 1990, p. 2). }\end{array}$
\end{tabular}

\begin{tabular}{lll}
\hline Sumanth & 1979 & "Productividad total- la razón de producción tangible entre \\
& insumos tangibles" (Sumanth, 1990, p. 2).
\end{tabular}

\begin{tabular}{lll}
\hline Bain 1982 & "Medida de lo bien que se han combinado y utilizado los \\
& recursos para cumplir los resultados específicos deseables" \\
& (Bain, 1982, p. 3).
\end{tabular}

\begin{tabular}{lll}
\hline Mercado & 1998 & "Producto final del esfuerzo y combinación de todos los \\
& recursos humanos, materiales y financieros que integran una \\
& empresa" (Velásquez de Naime et al., 2012, p. 849).
\end{tabular}

\begin{tabular}{lll}
\hline Gutiérrez & 2006 & "Lograr mejores resultados considerando los recursos \\
& empleados para generarlos" (Velásquez de Naime et al., 2012, \\
& p. 849).
\end{tabular}

\begin{tabular}{ll}
\hline Medina & 2010 \\
& "Forma de utilización de los factores de producción en la \\
& generación de bienes y servicios para la sociedad" (Medina, \\
& 2010, p. 112).
\end{tabular}

\begin{tabular}{llll}
$\begin{array}{l}\text { Cuadrado } \\
\text { Maroto }\end{array}$ & y & 2012 & $\begin{array}{l}\text { "Resultado de la utilización de distintos factores para obtener un } \\
\text { determinado volumen de productos y servicios" (Cuadrado \& } \\
\text { Maroto, 2012, p. 48). }\end{array}$ \\
\hline $\begin{array}{llll}\text { Galindo } \\
\text { Ríos }\end{array}$ & $\mathbf{y}$ & 2015 & $\begin{array}{l}\text { "Forma de medir la eficiencia con la que se utiliza el trabajo y } \\
\text { el capital para la producción de valor" (Galindo \& Ríos, 2015, } \\
\text { p. 2). }\end{array}$
\end{tabular}

Fuente. Elaboración propia con base a información a los autores citados en la tabla.

En la Tabla 1 es posible distinguir la evolución que ha presentado el concepto de productividad. Dicho pensamiento presenta una primera transformación, en donde los expertos en el tema empiezan a referir términos como recursos e insumos y la relación que existen entre ellos entendida como una razón, producto, medida, etc. Esto va también de la mano con las necesidades que el desarrollo económico exige, ya que es a mediados del siglo XX cuando se 
percibe un concepto más fundamentado y complejo, en donde no solo se habla de una relación básica entre los recursos empleados y el producto final, sino la interacción de diferentes elementos que impactan directamente en los niveles de productividad. Es esta nueva cualidad la que permite incorporar a las organizaciones de servicios dentro de este terreno, al tener un peso significativo en la generación de valor de las economías mundiales, haciendo su estudio relevante.

En síntesis, se puede señalar que la productividad está conformada por el número de recursos empleados (inputs) y el producto del bien o servicio generado (outputs). Al tener claro este concepto, se puede vislumbrar su importancia, la cual se centra en los procesos productivos, lo que significa optimizar los recursos empleados para la generación de un bien o servicio (González \& Carro, 2012).

Se hace especial énfasis que una reducción en los niveles de productividad tiene como resultado un incremento de los costos y por lo tanto un incremento en el precio final (Velásquez de Naime \& Rodríguez-Monroy, 2014); una disminución en la productividad puede estar asociado a diversos factores. Como parte de la literatura se pueden encontrar aquellos que impacta de forma directa a la productividad; no obstante, dependiendo del punto de vista del autor es la clasificación que se da, como se visualiza en la Tabla 2.

Tabla 2 .

Factores de la productividad

Autores Factores Elementos

\begin{tabular}{lll}
\hline Sumanth (1990); & Internos & Gestión administrativa; mano \\
Schroeder (1994); & de obra; materiales y \\
Prokopenko (1987); & suministros; maquinarias y \\
& equipos; métodos de trabajo; \\
& capital; cultura.
\end{tabular}

Fernández, Avella y Fernández (2003);

Acevedo (2004);

Externos

Ramírez (2005);

Anaya (2000);

Mosley, León y Pietri. (2005)
Reglamentación del gobierno; competencia, el cliente; política económica del gobierno; infraestructuras; estabilidad política; sociedad; entorno político; medio ambiente.

\begin{tabular}{ll}
\hline Levitan y Werneke (1984) & Económica \\
& la demanda; inversión en cambio cíclico en \\
& nuevas plantas y equipo; \\
& desarrollo de nuevas \\
& tecnologías y calificación y
\end{tabular}


experiencia de la fuerza de trabajo.

Institucional Rol de la conducta, actitudes e interacciones entre los principales participantes económicos.

Marvel, Rodríguez y Núñez (2011) Individuales

Grupales

Organizacionales
Motivación, competencias, satisfacción e identificación, compromiso e implicación.

Cohesión, participación. conflicto, 
La productividad puede ser referida a cualquier actividad económica; siendo más entendible para aquellas organizaciones generadoras de bienes, puesto que el producto es tangible y puede ser cuantificado. Sin embargo, cuando se trata de llevar este concepto al plano de los servicios resulta más complejo delimitarla y generalizarla debido a la naturaleza intangible de sus productos y a las múltiples actividades que se desarrollan (Maroto, 2013). En años recientes, las organizaciones prestadoras de servicios o terciario han cobrado especial relevancia; sin embargo, dichas investigaciones aún continúan siendo insuficientes, al ser percibidas esta tipología de organizaciones como poco productivas, aun cuando su importancia en la dinámica de la economía mundial es incuestionable (Maroto, 2013).

De acuerdo con Martínez (1992), Jean Fourastié fue uno de los primeros en estudiar la productividad en los diversos ámbitos económicos, agrupando así todas las múltiples actividades de las organizaciones de servicios en un único rubro. Del mismo modo, González, Del Río y Domínguez (1989), refieren que las organizaciones que pueden ser consideradas dentro del sector de servicios son aquellas en la que su principal actividad no es la producción de un bien.

Otro estudio sobre las organizaciones del sector terciario es la que propone Fisher (1939), quien menciona que la actividad que se genera es aquella que tienen que ver con productos inmateriales. Clark (1940) por su parte puntualizó el término de sector servicios y su relación con las múltiples actividades que se desarrollan; destacando la mención que da sobre la baja productividad que se presenta en este tipo de organizaciones.

En años recientes se puede advertir la evolución que ha presentado este concepto; ejemplo de esto se puede retomar de Romero, Toledo \& Ocampo (2014), quienes hacen una distinción entre la clásica definición del sector de servicios (toda actividad que no se desarrolla por parte de los sectores primario y secundario) y las recientes tendencias a incluir y resaltar la finalidad del producto (si es de uso final o intermedio).

Cabe destacar lo referido por Martínez (1992),el cual menciona que actualmente agrupar todas las actividades del sector servicios en la actualidad puede que no sea la mejor opción, ya que la diversidad y complejidad del sector obliga a realizar una distinción entre las diferentes actividades que se llevan a cabo. La importancia de conocer la amplia gama de actividades que se desarrollan dentro del sector de servicios, permite comprender la magnitud de su impacto dentro de cualquier economía.

Romero, Toledo \& Ocampo (2014), retoman la clasificación realizada por Browning y Singelman, en la cual clasifican en ocho apartados las diferentes actividades que se llevan a cabo en el sector terciario:

1. Servicios infraestructurales de conexión general

2. Servicios comerciales

3. Servicios auxiliares a bienes de conservación

4. Servicios financieros de circulación del capital

5. Servicios especializados a empresas comerciales y financieras

6. Servicios sociales

7. Servicios generales de la administración pública

8. Servicios personales 
Como se puede distinguir, la idea de heterogeneidad desarrollada por Fourastié es incompatible con la realidad que se vive actualmente, por lo que se corre el riesgo de que bajo esta perspectiva se tenga una visión sesgada de las organizaciones terciarias, en donde se refuerza la imagen de ser poco productivas.

A pesar de la importancia que está cobrando el sector servicios dentro de la comunidad científica, aún existen muchas áreas en la cuales se puede trabajar, sobre todo si se habla de las organizaciones de servicios sociales públicas, como lo son los institutos de educación públicos o privados en cualquiera de sus niveles, sanidad pública o privada y cualquier otra organización que proporcionen servicios sociales (Martínez, 1992; Maroto, 2013).

Son precisamente las organizaciones que brindan servicios sociales sobre las que se presta mayor atención, ya que es en esta clasificación donde se encuentran las organizaciones de educación, tanto públicas como privadas. En este caso se estudiará la productividad académica en Instituciones de Educación Superior (IES) de México.

De acuerdo con las cifras oficiales que publica el Instituto Nacional de Estadística y Geografía (INEGI, 2019) para México en el año 2018 el sector de terciario es el que más aportó al PIB, con el 66\% que se traducen $\$ 46,865,705.061$ millones de pesos. Dentro de este sector el apartado de educación contribuye con 2,731,624.408 millones de dólares, que es el 6\%. Estas cifras nos dejan claro la importancia del sector dentro de la economía de México; sin embargo, que el servicio de educación se encuentre en el décimo lugar habla de un área de oportunidad que no se ha aprovechado de la mejor forma y que si se implementan acciones que vengan desde dentro y fuera de las organizaciones educativas se puede lograr resultados importantes para la sociedad y economía mexicana.

\section{Productividad académica en la educación superior y sus determinantes}

Como se mencionó en un inicio, la importancia que tiene el estudio de la productividad académica en la educación superior se fundamenta porque es en este nivel educativo donde se llevan a cabo procesos cognitivos más complejos, a diferencia de la educación básica y media superior en donde se generan las herramientas elementales para el quehacer diario, como aprender a leer y escribir (UNESCO, 2015). Es en este nivel donde se originan nuevos conocimientos, desarrollos tecnológicos y científicos como lo señala la Subsecretaría de Educación Superior (SES, 2019).

Góngora (2010), menciona que cubrir con el ideal que se tiene del académico perfecto resulta difícil debido a la multifuncionalidad que conlleva esta profesión, si se le añade el hecho de que son los productos desarrollados por la investigación los que generan mayores distinciones, entonces el concepto de productividad académica tiende a ser asociada únicamente a la generación o aplicación innovadora del conocimiento. Asimismo, es relevante mencionar que la productividad académica es un elemento clave que permite a la IES obtener la notoriedad en el ámbito académico (Joy, 2009).

Si bien es cierto que existen diversas definiciones relacionadas a la productividad académica, no existe acuerdo alguno sobre el concepto como tal. Para Dundar y Lewis (1998), refieren que cuando se habla de educación superior la productividad presenta como característica principal su multidimensionalidad, esto como consecuencia de las diversas actividades desarrolladas.

García-Cepero (2010), menciona que la productividad académica presenta una fuerte relación entre los académicos universitarios y las responsabilidades que tienen dentro de su institución 
académica. Por otra parte, Martínez y Coronado (2003), señala que la productividad académica se compone de los resultados generados por los trabajadores de un departamento académico en relación a las actividades de investigación, docencia y extensión.

Munévar y Villaseñor (2008), menciona que la productividad dentro del marco académico es la relación que existe de los recursos usados en docencia, investigación y extensión y los productos que se generaron por cada una de estas actividades.

De acuerdo a lo que refiere Williams (2003), la productividad académica puede ser definida como aquella investigación académica que genere un aporte significativo, el cual, permita una mejor comprensión de cierta disciplina, dicha investigación académica es llevada a cabo por algún trabajador que sea parte de la facultad.

Es notorio que el concepto de productividad académica es relativamente nuevo y que se encuentra dentro de un proceso de construcción, por lo cual, se hace difícil señalar con precisión lo que se entiende como productividad académica, sin embargo, como un esfuerzo de conjuntar los conceptos anteriores se puede definir como aquella generación de productos académicos, asociados a la publicación de artículos científicos, patentes, dirección de tesis, convenios de colaboración, entre otros, resultado de las actividades de generación y aplicación del conocimiento y docencia desarrolladas por los PTC en las diversas áreas académicas en las que se desempeña.

Es importante remarcar la distinción que existe entre las actividades que desarrolla un PTC en México y en otros países. Lo anterior con la finalidad de comprender de mejor forma la naturaleza de la productividad académica; ya que en algunos países el PTC se dedica a actividades de investigación, en cambio, en países como en México, se focaliza en cuatro actividades fundamentales: docencia, tutoría y dirección individualizada del alumno, gestión académica y generación y aplicación del conocimiento (Secretaria de Educación Pública, 2019).

Esta característica de los PTC mexicanos hace que las actuales interpretaciones sobre la productividad académica se encuentren sesgadas a únicamente dos actividades (investigación y docencia), lo cual tiene un impacto significativo al momento de realizar mediciones internacionales y hacer comparaciones entre la productividad académica por las IES en México y las de otros países.

Dentro de la literatura es poco común encontrar investigaciones sobre la productividad académica en la educación superior, pero es aún más complicado para el caso de México, debido a la amplia gama de actividades que deben realizar los PTC. No obstante, se han encontrado aproximaciones teóricas y metodológicas importantes, como se presentan en la Figura 1. 


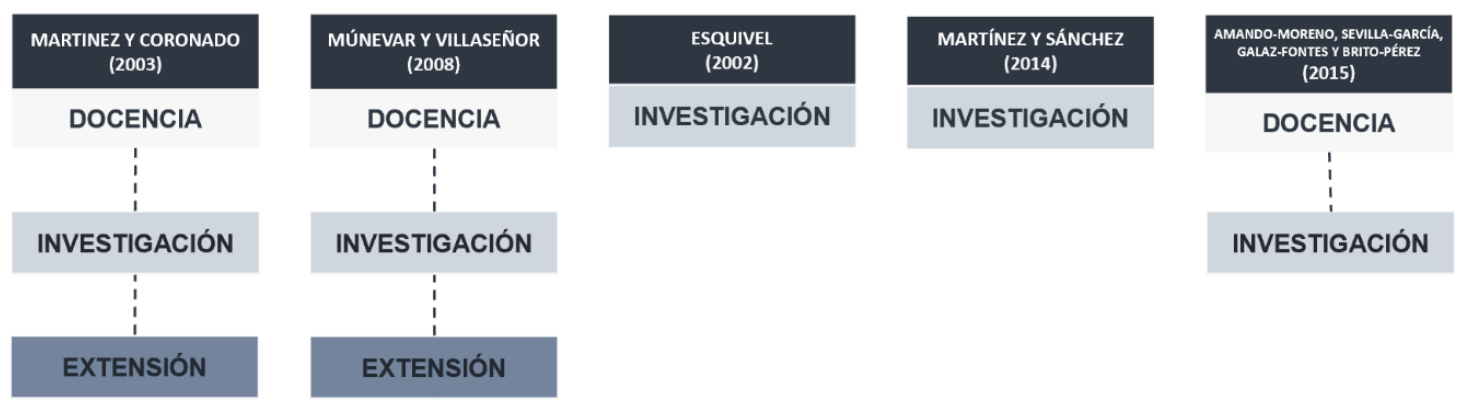

Figura 1. Productividad académica en la educación superior en México

Autores como Martínez \& Coronado (2003) y Munévar \& Villaseñor, (2008), llevaron a cabo una investigación sobre la productividad académica dentro del ámbito universitario mexicano. Para ellos la productividad académica se entiende como la relación entre las actividades de docencia, investigación y extensión; en dichas investigaciones respectivamente se enfatiza la necesidad de ahondar en la conceptualización de productividad académica, así como su aplicación en la sociedad a la cual pueda beneficiar. Para Amado-Moreno et al. (2015), retoman en sus respectivos trabajos únicamente las actividades de investigación y docencia; en donde resalta el aumento de horas frente a grupo que se tiene en la actualidad en comparación a las que se tenían a principios de los años 90, lo cual significa que el PTC tiene menos tiempo para dedicarle a las actividades de investigación.

Finalmente investigadores como Esquivel (2002) y Martínez \& Sánchez (2014), resaltaron únicamente el alcance e impacto que tiene la investigación en las diversas esferas académicas en México. Aun cuando solo toman en cuenta la investigación, en ambos estudios únicamente presenta resultados relacionados a los artículos publicados, que presenten un factor de impacto importante. Es claro que para el marco educativo superior mexicano existe una visión sesgada de lo que pude considerarse productividad académica debido a que dejan a un lado actividades como gestión académica y tutorías, las cuales son parte de las actividades obligatorias que desarrolla el PTC. Con esto en mente, es necesario acotar las actividades que desarrolla el PTC para comprender el fenómeno de la productividad académica, y no confundirla con la productividad científica, la cual está ligada únicamente a la investigación.

Lo anterior cobra importancia por las consecuencias que tiene la inversión de tiempo, recursos y esfuerzos dedicados a una o más actividades y los productos que de estas se generan, en demérito de alguna(s) de ella(s). A esto último se suma la indeterminación por parte de una institución internacional de peso significativo, como pudiera ser la UNESCO que puntualice de forma clara y precisa sobre las actividades que debe desarrollar un PTC a diferencia de un docente que solo se dedica a impartir clases.

Es importante resaltar que el presente estudio no tiene como objetivo demeritar el valor que tiene las actividades de gestión académica o tutoría, en especial si existen estudios donde resaltan la importancia de las tutorías como un elemento fundamental para la realización de un trabajo final de calidad (tesis) así como de formación profesional del alumno en el ámbito de investigación (Cruzata-Martínez, Bellido García, Velázquez-Tejeda, \& Alhuay-Quispe, 2018); por el contrario, es tratar de aterrizar el concepto de productividad académica a una realidad más universal.

Como se ha mencionado con antelación la productividad en términos simples refiere a una relación entre insumos y productos (inputs). En este sentido la productividad académica 
contempla aquellos productos desarrollados en las universidades por parte de los PTC en sus actividades académicas, en relación a sus determinantes. No obstante, se puede observar la presencia de dos posturas predominantes, por un lado, aquellas donde únicamente se toma en cuenta los productos relacionados a publicaciones $\mathrm{y}$, por otra parte, las que refieren a una amplia diversidad de productos asociados a las diferentes funciones o actividades académicas que realizan.

Prueba de lo anterior se puede encontrar en los trabajos de Martínez \& Coronado (2003), quienes resaltan la importancia de la productividad académica en las diversas actividades del PTC relacionadas con la investigación, docencia y extensión; en cambio autores como López, GarcíaCepero, Aguilar, Silva \& Aguado (2010), retoman únicamente los artículos publicados asociados a la investigación. En la Tabla 3 se presenta una recopilación de los determinantes de cada una de las actividades asociadas a la productividad académica planteados por diversos autores.

Tabla 3.

Determinantes de la productividad académica

Actividad y Autores Determinante

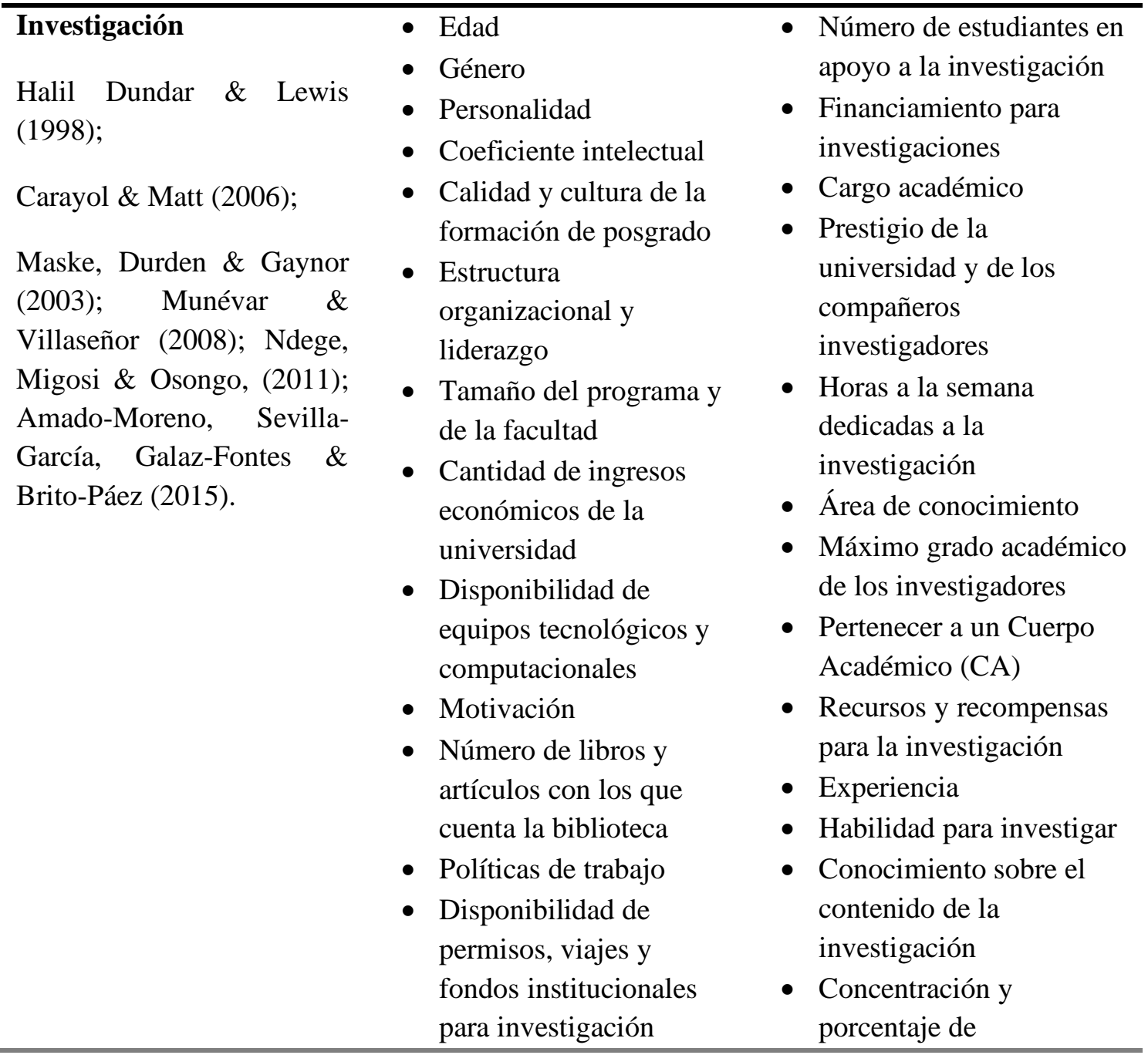


- Control institucional sobre el desempeño de la investigación (universidad pública o privada) publicaciones del profesorado.

- Porcentaje de profesores de tiempo completo

- Porcentaje de profesores con apoyo de investigación.

- Proporción de estudiantes graduados por facultad.

Docencia
Munévar \& $\quad$ Villaseñor
(2008);

- Número de cursos

- Número de alumnos

- Porcentaje de tiempo dedicado a la docencia.

Amado-Moreno, Sevilla-

García, Galaz-Fontes \&

Brito-Páez (2015).

\begin{tabular}{llll}
\hline Extensión & & - Número de horas semanales con organizaciones públicas \\
Munévar \& Villaseñor & - Relaciones académicas concretadas \\
(2008). & & & Tipo de actividad extrainstitucional
\end{tabular}

Fuente. Elaboración propia con base a información de los autores citados en la tabla

Como se puede apreciar en la Tabla 3, la actividad que más se ha estudiado es la de investigación. Diversos estudios como los de Dundar \& Lewis (1998), Carayol \& Matt (2006), Maske et al. (2003), Joy (2009), entre otros, han señalado que los productos como artículos científicos tienen un mayor impacto académico que cualquier otro producto, como parte de la función de investigación.

Al tener en cuenta la evidencia empírica sobre la productividad académica y conforme a las cuatro funciones que se determinan en México según Prodep se puede identificar que los determinantes que son de suma importancia para todas las actividades (docencia, investigación, gestión y tutoría) son edad, género y experiencia; de forma particular, para docencia: número de horas dedicadas a la actividad docente (horas clase, preparar las clases, etc.); investigación número de horas dedicadas a la actividad de investigación, área de conocimiento, máximo grado académico del investigador, financiamiento, disponibilidad de equipos tecnológicos y computacionales y políticas de trabajo; gestión académica: número de horas dedicadas a la actividad gestión y la disponibilidad de vinculación con organizaciones externas; por último tutorías: número de horas dedicadas a esta actividad, número de alumnos e infraestructura.

\section{El argumento de la discusión sobre la productividad académica}

Después de haber presentado una revisión sobre la productividad académica en términos teóricos y la evidencia empírica, se puede señalar que hablar sobre la productividad por sí sola, conllevaría a dejar a un lado el impacto que tiene en diversos aspectos de la producción. Si se analiza de forma más detallada, se tiene que la productividad va más allá de un estricto valor numérico, al 
final su impacto real se ve reflejado en un aumento en la calidad de vida de los individuos de una sociedad, tal y como lo refieren González \& Carro (2012); López et al. (2017); Velásquez de Naime \& Rodríguez-Monroy ( 2014), entre otros.

Medina (2010), menciona que en términos simples incrementar la productividad, es lograr hacer más con el mismo o menor esfuerzo, sin embargo, para lograr esto es necesario optimizar el uso de los recursos con los que se cuenta, como lo manifiesta. La bondad del concepto de productividad es que permea en diversas aspectos y niveles del quehacer humano, en coincidencia con Bain (1982), desde un punto de vista macro se refleja en el aumento del PIB; a nivel organización se traduce en un aumento en las ganancias e incluso en lo individual se refleja en el aumento de la calidad de vida de las personas.

Como se ha señalado a lo largo del documento, la productividad es un concepto que se adapta de forma más precisa a la producción de bienes, pese a esto, estudiar la productividad desde la perspectiva del sector terciario o de servicios puede dar excelentes resultados, tales como un aumento en la generación de empleos y del PIB (Martínez \& Picazo, 2000). Este escenario puede ser posible siempre y cuando la productividad de servicios sea vista desde la perspectiva de la organización, ya que de este modo se podrán cubrir sus necesidades y características.

De acuerdo con Maroto (2013) y Martínez \& Picazo (2000), a pesar de que los números favorecen al sector terciario y que en la actualidad existen muchos países que basan su economía en este sector, aún impera el concepto erróneo sobre la baja o nula productividad de este sector, cuando es solo una pequeña parte de las actividades desarrolladas en el sector terciario las que no son productivas, lo anterior propicia la necesidad de generar nuevos trabajos de investigación, cómo el que aquí se discute, en donde se adapte el concepto de productividad a este sector de forma adecuada y sin forzar su análisis, como en su momento lo señaló Martínez (1992).

Es importante destacar lo mencionado por Llisterri et al. (2014), cuando puntualizan que los países desarrollados, a diferencia del resto, han reconocido la trascendencia de las organizaciones de servicio educativos en el proceso de desarrollo de sus países, por ello han visualizado a las universidades como una fuente de conocimiento que les provee posibles respuestas a diversos problemas que enfrenta en su entorno, destinando así diferentes tipos de recursos humanos y tecnológicos para su óptimo aprovechamiento. Así, el concepto de productividad académica cobra relevancia, no solo por el hecho de generarle un mayor prestigio a la institución educativa, de la cual surgen nuevos conocimientos, sino por el beneficio que genera a la sociedad, como lo expresara el estudio de Martínez y Sánchez en 2014.

La evidencia empírica de los diversos estudios referidos en la presente investigación, como los de Dundar \& Lewis (1998), Carayol \& Matt (2006); Maske et al. (2003), Munévar \& Villaseñor (2008), Ndege et al. (2011), Amado-Moreno et al. (2015), entre otros, dejan ver que en la mayor parte de los estudios la productividad académica es vinculada directamente con la investigación, dejando de lado o dando menor peso a las actividades de docencia, gestión y tutoría.

Bajo esta óptica, se han identificado diversos determinantes relacionados con la productividad académica, los cuales se pueden clasificar como: individuales (aquellos que son inherentes al PTC como la experiencia, edad, género, área de conocimiento, etc.), colectivos (son los atributos con los que cuenta el cuerpo académico al que pertenece el PTC, como el prestigio, los recursos financieros) e institucionales (elementos que son provistos por parte de las universidades a los PTC para que desarrolle sus funciones adecuadamente). 
Para México, el concepto de productividad académica, se vuelve aún más complejo y ambiguo, contrastando incluso con lo establecido internacionalmente. Parte de esta problemática se encuentra asociada a las funciones que debe desarrollar el PTC en una universidad, las cuales van acorde a las reglas de operación que publica Prodep, en cuanto a docencia, tutoría, gestión académica y generación y aplicación del conocimiento. Estas funciones de acuerdo con las reglas de operación 2019 están orientadas a cubrir las metas planteadas en la Agenda 2030 de los Objetivos de Desarrollo Sostenible (ODS) presentados en el año 2015 por la UNESCO; sin embargo, dichas actividades no empatan con las recomendaciones planteadas por el Comité Mixto de Expertos sobre la aplicación de las Recomendaciones relativas al personal docente (CEART, 1997), en donde refieren que es necesario contar con las condiciones laborales óptimas para el desarrollo de actividades relacionadas a docencia, investigación y extensión.

Al momento existir una diversificación de las actividades que desarrolla el PTC; se debe tener claro un par de consideraciones: por un lado, si se incluyen las cuatro actividades mencionadas por prodep la comparación internacional sería poco factible ya que no se estaría midiendo bajo los mismos parámetros y el resultado que se pueda obtener podría ser parcial; por otro lado, si se opta por no incluir las actividades de tutorías y gestión académica, como generalmente ocurre al momento de estudiar este fenómeno, se tendría una visión sesgada de la realidad observada.

En síntesis, en el documento se ha podido argumentar sobre la inconsistencia entre la fundamentación teórica y la evidencia empírica sobre la productividad académica en las universidades mexicanas; lo cual dispersa su interpretación en demérito de una mayor precisión en su estimación y comparativo con la de otras instituciones dentro y fuera del país, cumpliendo así con el objetivo de discusión del presente documento.

\section{Conclusiones}

A partir de lo discutido en el presente documento, se puede puntualizar que inicialmente la productividad se apreciaba como la acción que permitía hacer más con una menor inversión. No obstante, en la actualidad es un elemento imprescindible para toda organización, a razón de que, si el objetivo es conseguir altas ganancias, es necesario ser productivo, si se logra esto último, se puede decir que la organización tiene las herramientas necesarias para competir, sin importar cual sea la actividad que desarrolle.

Es vital comprender que la productividad en el sector servicios no puede ser conceptualizada bajo la misma lógica que en el sector de bienes; debido a su naturaleza múltiple, ya que se podría caer en una imprecisión o equivocación al momento de tomar decisiones; por lo que es necesario realizar su adaptación conforme a las características propias de la organización.

En este punto, no se puede negar el impacto que tienen los servicios dentro de cualquier economía; como en este caso las organizaciones educativas, en específico las de educación superior, las cuales tienen un peso significativo en el desarrollo económico global. Lo anterior, porque es en estas donde se generan procesos de innovación, así como desarrollo científico y tecnológico, que a través del tiempo generan resultados positivos en el bienestar común de la sociedad en su conjunto.

Para el caso de México, es de vital importancia que las instituciones acoten y estandaricen la interpretación sobre el concepto de productividad académica, evitando con ello, la ambigüedad en las actividades que deben desempeñar los PTC en las universidades. Sobre todo, considerando 
que las actividades de investigación y docencia, son las más aceptadas dentro del campo científico, con apego a las recomendaciones internacionales. Si la productividad académica es entendida como la relación entre los productos académicos que son desarrollados en las actividades de investigación y docencias por parte de los PTC y los recursos institucionales empleados para su elaboración, entonces las funciones para los PTC deberían ser las mismas en cualquier institución de educación superior e independiente al lugar, permitiendo una comparación más objetiva entre ellas.

Derivado de todo lo anterior, se presenta un reto interesante, como es el de llevar a cabo un estudio comparativo sobre la productividad académica actual en las instituciones de educación superior en México ante instituciones de otros países, que únicamente contemple las actividades de investigación y docencia dentro de sus obligaciones; con el fin de observar el comportamiento de este fenómeno cuando existe una mayor la diversificación de actividades.

\section{Referencias}

Amado-Moreno, M., Sevilla-García, J., Galaz-Fontes, J., \& Brito-Páez, R. (2015). Análisis preliminar de la productividad académica en los institutos públicos tecnológicos mexicanos. Revista Iberoamericana de Educación Superior, 4(11), 125-135. https://doi.org/10.1016/s2007-2872(13)71936-x

Bain, D. (1982). Productividad. La Solución a los Problemas de la Empresa (primera ed). México: McGraw-Hill.

Bonilla, E. (2014). La importancia de la productividad como componente de la competitividad. Fundación Universidad de América, 6. Recuperado de http://repository.uamerica.edu.co/bitstream/20.500.11839/732/1/41584611-2012-2-EF.pdf

Carayol, N., \& Matt, M. (2006). Individual and collective determinants of academic scientists' productivity. Information Economics and Policy, 18, 55-72. https://doi.org/10.1016/j.infoecopol.2005.09.002

Comité Mixto de Expertos sobre la aplicación de las Recomendaciones relativas al personal docente (CEART). (1997). Recomendación relativa a la condición del personal docente de la enseñanza superior. Recuperado el 4 de octubre de 2019, de https://unesdoc.unesco.org/ark:/48223/pf0000113234/PDF/113234qaab.pdf.multi.page=40

Cruzata-Martínez, A., Bellido García, R., Velázquez-Tejeda, M., \& Alhuay-Quispe, J. (2018). La tutoría como estrategia pedagógica para el desarrollo de competencias de investigación en $\begin{array}{lllll}\text { posgrado. } & \text { Propósitos } & y & \text { Representaciones, } & 6(2),\end{array}$ https://doi.org/10.20511/pyr2018.v6n2.252

Cuadrado, J., \& Maroto, A. (2012). El problema de la productividad en España: Causas estructurales, cíclicas y sectoriales. Madrid: Fundación de las Cajas de Ahorros. Recuperado de http://www.funcas.es/publicaciones/Sumario.aspx?IdRef=9-08013

Domínguez, J. (2017). El desafío metodológico de la medición de la productividad. eXtoikos, 4950. Recuperado de https://dialnet.unirioja.es/servlet/articulo?codigo $=6108224$

Dundar, H., \& Lewis, D. (1998). Determinants of research productivity in higher education. Research in Higher Education, 39, 607-631. https://doi.org/10.1023/A:1018705823763 
Esquivel, G. (2002). Producción científica e impacto de los economistas académicos en México. Economía Mexicana. Nueva Época, XI(1), 5-30. Recuperado de http://www.economiamexicana.cide.edu/num_anteriores/XI1/01_GERARDO_ESQUIVEL.pdf

Felsinger, E., \& Runza, P. (2002). Productividad: Un estudio de caso en un departamento de siniestros. Universidad del CEMA.

Galindo, M., \& Ríos, V. (2015). Serie de Estudios Económicos. México ¿cómo vamos?, 1, 1-9. Recuperado de https://scholar.harvard.edu/files/vrios/files/201508_mexicoproductivity.pdf

García-Cepero, M. (2010). El estudio de productividad académica de profesores universitarios a través de análisis factorial confirmatorio: el caso de psicología en Estados Unidos de América* The Study of Faculty Productivity through Confirmatory Factor Analysis: the case of Psycho. Universitas Psychologica, 9(1), 13-26. Recuperado de http://www.redalyc.org/pdf/647/64712156002.pdf

Góngora, E. (2010). Dilemas éticos en la profesión académica contemporánea frente al marco dominante de reconocimientos a la productividad. Reencuentro, (57), 24-33. Recuperado de http://www.redalyc.org/articulo.oa?id=34012514004

González, M., Del Río, C., \& Domínguez, J. (1989). Los servicios: concepto, clasificación y problemas de medición. Ekonomiaz: Revista vasca de economía, 10-19. Recuperado de http://dialnet.unirioja.es/servlet/articulo?codigo $=1317365$

González, R., \& Carro, D. (2012). Administración Operaciones de las Productividad y Competitividad. Universidad Nacional de Mar del Plata, 18.

Instituto Nacional de Estadística y Geografía. (2019). Producto interno bruto trimestral, base 2013. Recuperado el 7 de septiembre de 2019, de https://www.inegi.org.mx/app/indicadores/?tm=0\#divFV493624493621

Joy, S. (2009). ¿Qué debo hacer y cómo debo hacerlo?: productividad académica de los psicólogos académicos. Boletín de psicología, (97), 93-116. Recuperado de https://www.uv.es/seoane/boletin/previos/N97-6.pdf

Labra, A. (2003). El comercio de servicios en el entorno de la globalización. Perfiles Educativos, 25, 69-83. https://doi.org/10.32399/rdk.12.23.638

Llisterri, J., Gligo, N., Homs, O., \& Ruíz-Devesa, D. (2014). Educación técnica y formación profesional en América Latina: el reto de la productividad. Caracas: CAF: Serie Políticas Públicas y Transformación Productiva 13. Recuperado de http://scioteca.caf.com/handle/123456789/378

López, A., Niembro, A., \& Ramos, D. (2017). Las empresas de servicios en América Latina: Un estudio exploratorio sobre factores de competitividad internacional, obstáculos y políticas públicas. TEC Empresarial 7, 11, 7-22. Recuperado de https://www.scielo.sa.cr/scielo.php?script=sci_abstract\&pid=S1659$33592017000100007 \& \operatorname{lng}=$ en\&nrm=iso\&tlng=es

López, W., García-Cepero, M., Aguilar, M., Silva, L., \& Aguado, E. (2010). Panorama general de la producción académica en la psicología iberoamericana, 2005-2007. Papeles del 
Psicólogo,

31 ,

296-309.

Recuperado

de

http://www.redalyc.org/articulo.oa?id=77815136009

Maroto, A. (2013). Las relaciones entre servicios y productividad: Un tema a impulsar en el ámbito regional y territorial. Investigaciones Regionales, 157-183. Recuperado de http://www.redalyc.org/pdf/289/28928823007.pdf

Martínez, J. (1992). Productividad de los servicios. Distribución y Consumo, 34-37. Recuperado de

https://www.mapa.gob.es/ministerio/pags/biblioteca/revistas/pdf_DYC/DYC_1992_5_co

mpleta.pdf

Martínez, J., \& Picazo, A. (2000). La productividad en los servicios. ICE Sector Servicios, 127140.

Martínez, M., \& Coronado, G. (2003). Indicadores para la evaluación integral de la productividad académica en la educación superior. RELIEVE - Revista Electronica de Investigacion y Evaluacion Educativa, 9(1), 45-72. Recuperado de https://www.uv.es/RELIEVE/v9n1/RELIEVEv9n1_2

Martínez, N., \& Sánchez, I. (2014). Productividad investigadora en las universidades públicas mexicanas: área de administración, 2000-2013. NovaRua. Revista Universitaria de Administración, IV(VIII), 37-52. Recuperado de http://erevistas.uacj.mx/ojs/index.php/NovaRua/article/view/21/23

Marvel, M., Rodríguez, C., \& Núñez, M. (2011). La productividad desde una perspectiva humana: Dimensiones y factores. Intangible Capital, 7, 549-584. https://doi.org/10.3926/ic.194

Maske, K., Durden, G., \& Gaynor, P. (2003). Determinants of scholarly productivity among male and female economists. Economic Inquiry, 41(4), 555-564. https://doi.org/10.1093/ei/cbg027

Medina, J. (2010). Modelo integral de productividad, aspectos importantes para su implementación. Revista EAN, $\quad$ (69), $110-119$. https://doi.org/10.21158/01208160.n69.2010.519

Munévar, D., \& Villaseñor, M. (2008). Producción de conocimientos y productividad académica. Revista de Educación y Desarrollo, 61-67. Recuperado de http://www.cucs.udg.mx/revistas/edu_desarrollo/anteriores/8/008_Munevar.pdf

Ndege, T., Migosi, J., \& Osongo, J. (2011). Determinants of research productivity among academics in Kenya. Education Economics and Development, 2(3), 288-300. Recuperado de https://onlinelibrary.wiley.com/doi/abs/10.1093/ei/cbg027

OIT. (2016). El Recurso Humano y la Productividad. Organización Internacional del Trabajo. https://doi.org/10.1016/S0305-750X(97)10057-2

Romero, J., Toledo, Y., \& Ocampo, V. (2014). El sector servicios: revisión de los aportes para su teorización y estudio. Tecsistecatl, 6(16), 1-20. Recuperado de http://www.eumed.net/rev/tecsistecatl/n16/sector-servicios.pdf

Secretaria de Educación Pública. Reglas de Operación del Programa para el Desarrollo 
Profesional Docente para el ejercicio fiscal 2019., Pub. L. No. 07/02/19, 1 (2019). México. Recuperado de

http://diariooficial.gob.mx/nota_detalle.php?codigo=5552805\&fecha=13/03/2019

Subsecretaría de Educación Superior. (2019). La innovación tecnológica y científica que realicen las instituciones y demás organismos con sostenimiento del gobierno deben tener como prioridad el bienestar de las mexicanas y los mexicanos. Ciudad de México. Recuperado de https://educacionsuperior.sep.gob.mx/pdfs/difusion/difusion09082019.pdf

Sumanth, D. (1997). Ingeniería y Administración de la Productividad (Primera Ed). Estado de México: Mc Graw-Hill/Interamericana de México S.A. de C.V.

UNESCO. (2015). Informe de Seguimiento de la ETP en el Mundo. Recuperado de http://www.educandoenigualdad.com/wpcontent/uploads/2015/05/EPT2015.compressed.pdf

UNESCO. (2017). Desglosar el Objetivo de Desarrollo Sostenible 4. Educación 2030. Organización de las Naciones Unidas para la Educación, la Ciencia y la Cultura, 36. Recuperado de http://unesdoc.unesco.org/images/0024/002463/246300S.pdf

Velásquez de Naime, Y., \& Rodríguez-Monroy, C. (2014). Percepción de la Gerencia sobre los factores que afectan la productividad en la Pyme del sector metalúrgico y minero de Venezuela. Interciencia ISSN:, 39, 704-711. Recuperado de http://www.redalyc.org/articulo.oa?id=33932433004

Velásquez de Naime, Y., Rodríguez, C., \& Guaita, W. (2012). Modelo de los factores que afectan la productividad. En J. C. Prado \& J. García (Eds.), Modelo de los factores que afectan la productividad (pp. 847-854). Congreso de Ingeniería de Organización. Recuperado de https://dialnet.unirioja.es/servlet/articulo?codigo $=4248489$

Williams, H. A. (2003). A Mediated Hierarchical Regression Analysis of Factors Related to Research Productivity of Human Resource Education and Workforce Development Postsecondary Faculty. The School of Human Resource Education and Workforce Development. Louisiana State University. 